\title{
Correction to: Split $\mathrm{N}$ and $\mathrm{P}$ addition decreases straw mineralization and the priming effect of a paddy soil: a 100-day incubation experiment
}

\author{
Dongdong Wang ${ }^{1,2} \cdot$ Zhenke Zhu $^{1} \cdot$ Muhammad Shahbaz $^{3,4} \cdot$ Liang Chen $^{5} \cdot$ Shoulong Liu $^{1} \cdot$ Kazuyuki Inubushi $^{6}$. \\ Jinshui $\mathrm{Wu}^{1,2} \cdot \mathrm{Tida}^{1} \mathrm{Ge}^{1}$
}

Published online: 6 August 2019

(C) Springer-Verlag GmbH Germany, part of Springer Nature 2019

Correction to: Biology and Fertility of Soils

https://doi.org/10.1007/s00374-019-01383-6

The author regret that the above article originally published with an error in the equation under "Calculation of GHG emission, PE, C-mineralization, microbial C use efficiency, and enzyme activity" section. The corrected equation is shown as follows:

$\mathrm{CUE}={ }^{13} \mathrm{C}-\mathrm{MBC} /\left({ }^{13} \mathrm{C}-\mathrm{MBC}+\mathrm{Rcum}\right)$

The remainder of the article remains unchanged.

Pulisher's note Springer Nature remains neutral with regard to jurisdictional claims in published maps and institutional affiliations

The online version of the original article can be found at https://doi.org/ 10.1007/s00374-019-01383-6

Zhenke Zhu

zhuzhenke@isa.ac.cn

1 Key Laboratory of Agro-ecological Processes in Subtropical Region \& Changsha Research Station for Agricultural and Environmental Monitoring, Institute of Subtropical Agriculture, Chinese Academy of Sciences, Changsha 410125, Hunan, China

2 University of Chinese Academy of Sciences, Beijing 100049, China

3 Department of Soil and Environment, Swedish University of Agricultural Sciences, Box 7014, 75007 Uppsala, Sweden

4 Centre for Environmental and Climate Research, Lund University, 22362 Lund, Sweden

5 Faculty of Life Science and Technology, Central South University of Forestry and Technology, Changsha 410004, Hunan, China

6 Graduate School of Horticulture, Chiba University, Matsudo, Chiba 271-8510, Japan 